\title{
Evidencias Psicométricas del Autoinforme del INSEBULL en Adolescentes de Dos Colegios de la Ciudad del Cusco
}

\author{
Psychometric Evidence of the INSEBULL Self-Report in Adolescents from Two Schools in the City of Cusco
}

Adriana Gabriela Loaiza Dargent ${ }^{1}$, Cecilia Patricia Castro Chávarry ${ }^{1}$

\section{RESUMEN}

El propósito de la presente investigación fue establecer algunas evidencias psicométricas del Autoinforme de la prueba INSEBULL, el cual tiene como objetivo identificar el grado en que los estudiantes expresan riesgo de verse involucrados en situaciones de acoso escolar o Bullying. La muestra fue de 471 estudiantes de primero a cuarto año de secundaria de un colegio privado y otro estatal de la ciudad del Cusco (Perú). En el estudio de validez semántica mediante la participación de 10 jueces, se halló que todos los ítems, excepto el 34, obtuvieron un valor de la V de Aiken de 0,80 o más. El análisis factorial confirmatorio estableció un modelo de seis factores. El alfa de Cronbach para la puntuación total de instrumento fue 0,873 y las escalas Intimidación, Victimización y Expresión de Temores Escolares presentan valores alfa mayores a 0,80, mientras que las escalas de Solución Moral, Falta de Integración Social e Identificación de los Participantes obtuvieron valores alfa menores a 0,60. Aunque no se halló una diferencia estadísticamente significativa entre las puntuaciones totales del colegio estatal y el privado, se establecieron baremos separados para cada uno de ellos, recomendándose precaución respecto al uso de dichos baremos, dada la baja consistencia interna de las puntuaciones de varias de las escalas del instrumento.

PALABRAS CLAVE: Bullying, vulnerabilidad, confiabilidad, análisis factorial, validez de contenido.

\section{SUMMARY}

The objective of the present study was to provide some psychometric evidences for the INSEBULL Self-Report, whose objective is to identify the measure to which students express a risk of getting involved in bullying. The sample consisted of 471 first to fourth high school students from a public and a private school in Cuzco (Peru). In the semantic validity study with 10 experts, it was found that all of the items, except the 34, had Aiken's V values of .80 or more. Confirmatory factor analysis established a six-factor model. Cronbach's alpha value for the total score was .873 and the factors of intimidation, victimization and expression of school fears had Cronbach's alpha values greater than .80 , while the factors of moral solution, lack of social integration and identification of the participants obtained values less than .60. Although significant differences between the total score of public and private schools was not found, we built separate norms for each of them, but at the same time, we advise to take care in using these norms, given the low internal consistency in the scores of several of the scales of this instrument.

KEYWORDS: Bullying, vulnerability, reliability, factor analysis, content validity.

Universidad Peruana Cayetano Heredia. Lima, Perú. 


\section{INTRODUCCIÓN}

El bullying o acoso escolar es un fenómeno que viene afectando el ámbito educativo nacional e internacional. Avilés (2002) lo define como el constante hostigamiento dirigido a un estudiante en desventaja social o física, de manera sistemática e intencional. Si el acoso escolar no es detectado a tiempo en las escuelas, es probable que cause gran daño psicológico y social en los involucrados, afectando no solo su desempeño académico, sino también su desarrollo futuro.

Los primeros estudios en relación a la violencia en los contextos educativos fueron realizados por Olweus en 1970 a raíz de diversos incidentes de agresión en las escuelas de Noruega. Para él, las conductas asociadas al fenómeno en cuestión son aquellas que dañan e intimidan a la víctima, recalcando la existencia de una relación de poder asimétrica, por lo que no deben confundirse con discusiones o juegos entre dos estudiantes con igual capacidad para defenderse (citado por Garaigordobil \& Oñederra, 2010). Para Avilés y Elices (2007), el bullying es un proceso de socialización a través del cual uno o varios alumnos (agresores), de manera intencional adoptan actitudes y conductas agresivas hacia otro compañero (víctima). Este maltrato se da de manera constante, a lo largo del tiempo, y en este fenómeno sobresale la diferencia de poderes siendo el objetivo dañar a la víctima por medio de conductas intimidantes (Ccoicca, 2010).

Autores como Avilés (2002) y Joffre et al. (2011), precisan que el acoso escolar puede observarse de cuatro formas dentro de las escuelas: física, verbal, psicológica y social. En primer lugar, el acoso fisico asume su forma directa cuando el agresor ejerce fuerza física sobre la víctima (empujones, golpes y patadas, entre otros), mientras que en su forma indirecta el agresor usa la fuerza para atentar contra los objetos personales de la víctima. Por otro lado, Joffre et al. (2011) afirman que el acoso verbal es el más frecuente; en su forma directa, la persona agredida se encuentra presente cuando los agresores la insultan, se burlan de ella, la humillan, etc. En la forma indirecta la víctima no está presente ni es consciente de que los agresores la descalifican o formulan rumores negativos sobre ella en su entorno social. En lo que respecta al acoso de tipo social, Avilés (2002) refiere que este se da de manera directa cuando se bloquea el acceso de la víctima al grupo de manera imperativa; en cambio, en la forma indirecta la víctima es ignorada constantemente y no es considerada dentro de las actividades del grupo de estudiantes. Finalmente, el acoso de tipo psicológico se caracteriza por atentar contra la autoestima de la víctima y producir en esta una sensación de miedo y vulnerabilidad. Es necesario recalcar que para Ccoicca (2010) este tipo de acoso está implícito en todos los demás.

\section{Características del Bullying}

De acuerdo con Castro-Morales (2011), para que pueda hablarse de bullying o acoso escolar, deben cumplirse al menos tres de las siguientes cinco características: a) La víctima se siente vulnerada o intimidada; b) La víctima se siente socialmente excluida; c) El agresor es percibido como más fuerte; d) La intensidad del abuso tiende a incrementarse y e) El abuso se produce en su mayoría en espacios ocultos. Las conductas asociadas al acoso escolar deben ser claramente diferenciadas de cualquier otro tipo de conflicto o agresión dentro del colegio y para ello Piñuel y Oñate (2005) proponen tres criterios diagnósticos. El primer criterio se refiere a que los actos de agresión en cualquiera de sus tipos deben caracterizarse por tener una naturaleza intencional, es decir que el agresor de manera consciente decide causar algún tipo de daño a la víctima. El segundo criterio consiste en que la víctima percibe que es atacada de manera sistemática; es decir, las situaciones de hostigamiento no son consideradas como eventos aislados sino como parte de un continuo maltrato que produce dolor sostenido en la víctima. Por último, el tercer criterio marca que el estudiante que es acosado se ve vulnerado y afectado en las diversas áreas de su vida; es decir, que perjudica su desarrollo social, académico, afectivo, etc.

\section{Roles Involucrados en el Bullying}

Avilés (2002), Enríquez y Garzón (2015) y Garaigordobil y Oñederra (2010) coinciden en que son tres los roles implicados en la dinámica de acoso escolar y se caracterizan por lo siguiente:

- Agresor. Los agresores, también conocidos como bullies, son los estudiantes que ejecutan las acciones violentas. Se caracterizan por ser impulsivos, dominantes y tener un bajo nivel de empatía. Suelen ser físicamente fuertes y tener una forma agresiva de resolver problemas.

- Víctima. Se refiere al estudiante que es intimidado o violentado. Puede presentar características como ansiedad, baja autoestima, timidez, vulnerabilidad física, limitadas habilidades sociales y depresión. Para Olweus (1998) es posible diferenciar entre dos tipos de víctimas: las pasivas y las provocadoras. 
El primer tipo se caracteriza por no defenderse de las agresiones y callar los abusos. En cambio, las víctimas provocadoras son aquellas que responden con conductas ansiosas y agresivas. Los alumnos hiperactivos o con problemas de concentración suelen caer en este perfil.

- Espectadores- testigos. Son aquellos estudiantes que observan las situaciones de acoso y que no realizan ningún tipo de acción para impedirlas. Según Olweus (1998) la probabilidad de que los espectadores sean partícipes de las agresiones y de que no las califiquen como negativas, aumenta cuando ocurren dos fenómenos. El primero es el "contagio social", es decir, el hecho de que los testigos tienden a minimizar la violencia ejercida hacia la víctima cuando tienen una percepción positiva del agresor. Por otro lado, el segundo fenómeno se conoce como "debilitamiento social", este se da cuando el control de impulsos de los observadores se ve mermado debido a la habituación y exposición de estos a conductas violentas.

\section{Factores de Vulnerabilidad al Bullying y Consecuencias}

Enríquez y Garzón (2015), Avilés (2002) y Castro-Morales (2011), plantean que los factores que influyen en la ocurrencia de acoso escolar pueden estar relacionados a los siguientes contextos: familiar, personal y escolar.

Contexto familiar. La falta de cuidado parental, escasa supervisión, medidas disciplinarias autoritarias, castigos físicos, baja cohesión familiar, entre otros, pueden relacionarse al desarrollo de actitudes agresivas en los escolares. Para Córdova et al. (2012), la violencia familiar, la situación socioeconómica y hogares monoparentales también son factores que favorecen el desarrollo de modelos agresivos de interacción. Piñuel (2007, citado por Amemiya et al., 2009) indica que existen ciertos mensajes transmitidos por los padres que pueden favorecer el desarrollo de dinámicas violentas en la escuela. Se trata de mensajes como: negar el acoso que el estudiante denuncia, normalizar la violencia, decir que esto también les ocurrió en la escuela, hacer parecer el abuso como positivo porque prepara para la vida y por último, incitar al estudiante a responder violentamente ante los ataques para defenderse.

Contexto personal. Córdova et al. (2012) señalan que las discapacidades físicas o mentales, baja autoestima, nivel socioeconómico, orientación sexual, contextura física y pertenencia a algunos grupos étnicos pueden ser factores de riesgo para convertirse en víctima de acoso escolar. De la misma manera, características como la inseguridad, pobre control de impulsos, incapacidad para resolver problemas y poca empatía, se relacionan con una mayor probabilidad de ser agresor.

Contexto escolar. Se resaltan tres características del colegio: el tamaño, la presencia de profesores y la organización interna. Mientras más grande es el colegio más difícil se hace controlar y vigilar las relaciones entre estudiantes, por lo que existen más espacios de riesgo que mantengan ocultas las conductas violentas. En segundo lugar, se resalta el rol de los profesores. Según Olweus (1998), a mayor número de docentes supervisando a los alumnos, menos son los incidentes de acoso. Por ello, es necesario que el centro educativo organice a los maestros para vigilar las interacciones entre estudiantes.

Para Díaz (citado por Enríquez \& Garzón, 2015) otros factores que pueden agudizar la presencia de situaciones de bullying dentro de las instituciones educativas son: la normalización del uso de la agresión como medio de solución de conflictos, la indiferencia de los profesores ante el acoso y la poca conciencia de las necesidades o características peculiares del alumnado. Asimismo, la televisión y el internet son fuentes constantes de estímulos violentos y la exposición prolongada de los estudiantes a este tipo de información puede generar en ellos el aprendizaje de conductas agresivas y la habituación a este modo de actuar.

Las consecuencias del bullying impactan de manera negativa en la vida de la víctima, pero también afectan a los agresores y espectadores porque impactan en sus patrones de socialización. Wolf y Esteffan (2008) identificaron que la víctima tiende a sufrir consecuencias como: depresión con ideación suicida, falta de confianza en sí misma, episodios de ansiedad con síntomas fisiológicos, enuresis, deserción escolar, aislamiento social, trastornos de alimentación, bajo rendimiento académico, vómitos e insomnio. García y Ascensio (2015) agregaron el tartamudeo, llanto sin razón, pesadillas frecuentes, desarrollo de trastornos fóbicos y conductas de negar lo que están pasando. Por otro lado, en el caso del agresor, las consecuencias se resumen en incurrir en conductas antisociales, abusar de sustancias y mantener el rol de agresor hasta la vida adulta. Asimismo, Voors (2012, citado por Enríquez \& Garzón, 2015) señaló consecuencias como poca 
capacidad para adaptarse, depresión, problemas con la autoridad, verse involucrados en problemas legales y fracaso escolar. Finalmente Garaigordobil y Oñederra (2010) mencionan que los espectadores tienden a interiorizar un aprendizaje social que conduce a percibir la violencia como un buen método para conseguir lo que se quiere o que su uso es natural. En algunos casos, el miedo forma parte de los recursos en los que justifican su falta de acción en favor de la víctima y, en general, se acostumbran a no ser solidarios con los demás, reduciendo se capacidad empática.

\section{Instrumentos para la Evaluación del Bullying}

En el Perú se han realizado diferentes investigaciones en torno al tema de acoso escolar, por lo que el uso de instrumentos para medirlo ha sido necesario. La revisión de información en torno al tema, ha permitido identificar el uso de los instrumentos que se reseñan a continuación.

Escala DEVIDA: Cobián et al. (2015) indican que la construcción de este instrumento formó parte del IV Estudio Nacional de Prevención y Consumo de Drogas en estudiantes de secundaria realizado en el Perú en el periodo 2012-2013. Este cuestionario fue diseñado por el equipo técnico de DEVIDA y fue usado en 47 ciudades del Perú con una muestra de 48,025 estudiantes. El informe final del estudio permitió observar resultados específicos en el área de acoso escolar en tres niveles: bajo, medio y alto. La escala DEVIDA consta de 14 ítems que se presentan como situaciones de acoso escolar que el estudiante pudo haber experimentado durante el último año (Comisión Nacional para el Desarrollo y Vida sin Drogas-DEVIDA, 2013).

Cuestionario de Intimidación entre Iguales: Este instrumento fue desarrollado en España por Ortega, Mora-Merchán y Mora (2006), está compuesto por 30 ítems y su objetivo es explorar las situaciones de acoso en la escuela. Según Sáenz (2010) este instrumento fue validado en Lima por Vivian Landázuri en el 2007 con la colaboración de 35 alumnos de un colegio privado del distrito de San Borja (Lima, Perú). Esta investigadora obtuvo un coeficiente alfa de Cronbach de 0,83 , pero no se reportaron datos sobre la validez de la prueba.

Autotest Cisneros de Acoso Escolar: Fue construido en España por Iñaki Piñuel y Araceli Oñate en el 2005. Se compone de 50 ítems los cuales están agrupados en 10 escalas. Proporciona un índice general de acoso y uno de intensidad. Asimismo, 8 de las 10 escalas están orientadas a evaluar las diversas formas en que se presenta la violencia en la escuela. Según Piñuel y Oñate (2005), la confiabilidad de esta escala reporta un alfa de Cronbach de 0,857. En lo que respecta a la validez de constructo, el método de análisis de ítem indica que todos los coeficientes de correlación son significativos y mayores a 0,30 .

Instrumentos para la evaluación del Bullying INSEBULL: Esta batería de pruebas fue desarrollada en España por Avilés y Elices (2007). Se compone de una prueba principal que vendría ser el Autoinforme, y dos opcionales: Heteroinforme-C (compañeros) y Heteroinforme-P (profesores). Los factores que son evaluados por el Autoinforme son los siguientes: intimidación, victimización, red social, solución moral, falta de integración social, constatación del maltrato, identificación de los participantes en el bullying y expresión de temores escolar. El heteroinforme para compañeros (Heteroinforme-C) evalúa las dimensiones de intimidación, victimización y contemplación. El heteroinforme para el profesorado (Heteroinforme-P) abarca las dos primeras dimensiones del Heteroinforme-C más el área de apoyo a la víctima. El INSEBULL cuenta con información detallada sobre los procesos estadísticos de su construcción y validación, los cuales se basan en más de una década de investigación sobre el tema de acoso escolar. En el Perú, las propiedades psicométricas del Autoinforme de la prueba INSEBULL fueron estudiadas por Pinedo (2015) y Rimaycuna (2015). El primero estableció la validez semántica de los ítems a través del criterio de jueces y halló valores $\mathrm{V}$ de Aiken mayores a .80 para todos los ítems. Para la validez de constructo, este autor realizó un análisis factorial confirmatorio, a través del cual identificó un modelo de ocho factores. También estableció la confiabilidad para la prueba en general y no por factores, obteniendo un alfa de Cronbach de 0,774 . Por su parte, Rimaycuna (2015) no estudió la validez semántica y al analizar la validez de constructo identificó un modelo de siete factores a través del análisis factorial confirmatorio, eliminando el factor de Vulnerabilidad escolar ante el abuso (Expresión de temores). El alfa de Chonbach fue .82 para el total de la prueba. Este autor tampoco analizó la confiabilidad por factor. En lo que respecta a investigaciones fuera del Perú, solo se halló un trabajo realizado por Olaya et al. (2014) en Colombia. Este estudio no se ocupó de estudiar la validez semántica pero sí de analizar la validez de constructo mediante un análisis factorial confirmatorio, a través del cual se identificó un modelo de ocho factores. En cuanto a la confiabilidad se halló 
un alfa de Cronbach de .911, pero tampoco reportaron valores de confiabilidad por factores.

Es necesario mencionar que, en comparación con las otras pruebas mencionadas anteriormente, el Autoinforme del instrumento INSEBULL cuenta con datos más detallados sobre su construcción y procesos estadísticos originales. Ello hace más fácil su validación en otros contextos. Por otro lado, esta prueba contempla dentro de sus ítems los tres criterios diagnósticos de acoso escolar: sistematicidad, diferencia de poderes e intencionalidad. Además, no solo dirige su atención hacia los estudiantes que son víctimas, sino que también cuenta con ítems y factores que se orientan a explorar los demás roles en las dinámicas de acoso escolar. Este aspecto podría facilitar la intervención e identificación de alumnos en riesgo dentro de los contextos escolares.

\section{MÉTODO}

\section{Participantes}

La muestra estuvo conformada por 471 alumnos, de los cuales 233 pertenecían a un colegio privado y 238 a un colegio estatal. Por otro lado, 253 estudiantes eran hombres. Cursaban el primer año de secundaria, 90 estudiantes, 91 estaban en segundo de secundaria, 125 en tercero de secundaria y 165 estudiantes cursaban el cuarto de secundaria. Las edades de los estudiantes fluctuaban entre los 11 y 15 años. El tamaño de muestra fue determinado siguiendo el principio planteado por Morales (2012) de contar con al menos 10 sujetos por cada ítem, lo que puede considerarse adecuado para llevar a cabo análisis multivariados (Catena et al., 2003). Teniendo en cuenta que la prueba consta de 33 ítems, se consideró que el tamaño muestral era adecuado.

\section{Instrumento}

Como ya se mencionó, el instrumento INSEBULL se compone de tres partes: Autoinforme, Heteroinforme-C (Compañeros) y Heteroinforme-P (Profesores). Su diseño posee un sólido marco teórico en el que se describe al bullying como un fenómeno caracterizado por sistematicidad, diferencia de poderes e intencionalidad.

Según los autores del instrumento, Avilés y Elices (2007), los antecedentes del INSEBULL se remontan a 1999, año en el que fue creado el Autoinforme a partir de la adaptación de dos pruebas: El Cuestionario sobre Intimidación entre Iguales y el Cuestionario sobre Abusos entre Compañeros. En un primer momento, esta herramienta contaba con 33 ítems y se llenaba de manera anónima. En el 2003 se agregó el Heteroinforme, por lo que dejó de ser anónima y se volvió confidencial debido a su carácter sociométrico, lo que requiere la identificación de los individuos en la dinámica de acoso. Asimismo, se agregaron tres ítems relacionados a la percepción de los alumnos sobre el rol que asumen durante las situaciones de bullying. Finalmente, la última versión se desarrolló en el 2005. En esta, la definición de bullying se mejoró a través de indicadores más claros y se formalizaron las propiedades psicométricas de los heteroinformes. La muestra para el estudio de esta versión fue de 2698 estudiantes de primero a cuarto de secundaria residentes en las ciudades españolas de Valladolid, Álava y Vizcaya.

El Autoinforme da como resultado la percepción global del estudiante sobre el riesgo de involucrarse o ser afectado por situaciones de bullying. De este modo, constituye una exploración de la vulnerabilidad ante el bullying. Este instrumento está constituido por un ítem cualitativo y otros 33 ítems cuantitativos; algunos de estos se estructuran para elegir solo una alternativa, mientras que en otros se puede marcar más de una. Los reactivos están repartidos en las siguientes ocho dimensiones:

- Intimidación: Se refiere al grado de conciencia del alumno para verse a sí mismo como agresor en situaciones de acoso.

- Victimización: Grado de conciencia en que el alumno se percibe como víctima en la dinámica de bullying.

- Red Social: Es la percepción que tiene el alumno sobre la dificultad que presenta para hacer amistades o establecer relaciones sociales positivas en su entorno escolar.

- Solución Moral: Ligada a dificultad para salir del maltrato y al juicio moral del sujeto ante esta situación.

- Falta de Integración Social: Se entiende como la dificultad para relacionarse con la familia, profesorado y compañeros.

- Constatación del Maltrato: Se refiere al grado de conciencia que los estudiantes tienen sobre las situaciones de maltrato y la causa de estas.

- Identificación de participantes: Ligado al grado de conocimiento que los estudiantes tienen sobre el maltrato.

- Expresión de temores escolares: Se entiende como 
la exteriorización de miedos relacionados al contexto educativo.

Para la calificación se cuenta con un CD de corrección que arroja resultados individuales y grupales. Los baremos se establecieron a través de puntuaciones típicas derivadas con media 100 y desviación típica de 15.

Las propiedades psicométricas de los instrumentos originales que componen el INSEBULL han pasado por procesos estadísticos formales realizados en España por Avilés y Elices (2007). La consistencia interna, a través del coeficiente alfa de Cronbach para el Autoinforme fue .84. En cuanto a la consistencia interna de cada uno de los factores del Autoinforme se halló que las dimensiones de Intimidación y Victimización presentan valores altos con un alfa de 0,90 y 0,89 respectivamente. Los factores de Solución Moral, Red Social, Falta de Integración Social y Constatación del Maltrato presentan una consistencia interna baja con valores de alfa entre 0,44 y 0,60 . Finalmente, los factores con menos consistencia interna son los de Identificación de participantes en el bullying $(0,29)$ y Vulnerabilidad escolar ante el abuso o Expresión de temores escolares $(0,04)$. Avilés y Elices (2007) mencionan que la baja confiabilidad en estas escalas se puede deber a que estas tienen menos cantidad de ítems.

Por otra parte, al estructurar el instrumento original, Avilés y Elices (2007) también analizaron dos tipos de validez: de contenido y de constructo. La validez de contenido del Autoinforme se determinó a través del criterio de jueces. Para esto, se contactó a 10 expertos a quienes se les solicitó valorar la adecuación de cada uno de los ítems. Los jueces estuvieron de acuerdo en que los ítems no solamente eran adecuados para la prueba en general, sino que también definían acertadamente cada una de las dimensiones del instrumento. La validez de constructo del Autoinforme se determinó a través del análisis factorial exploratorio con rotación Varimax. Para definir qué ítems conformaban cada factor se tuvo como criterio que cada reactivo tuviera un peso factorial igual o mayor a 0,30 , por lo que algunos de los ítems se encuentran hasta en dos factores. Este es el caso de los ítems 2, $5,8,9,14,26,27,32$ y 33 . Los resultados indicaron la existencia de ocho factores, lo cuales explicaron el $57.10 \%$ de la varianza. Los factores de Intimidación y Victimización presentaron ítems con cargas factoriales altas. En cambio, los demás factores tuvieron reactivos con cargas factoriales en niveles moderados y bajos.
Según Avilés y Elices (2007, p. 21) "el resto de factores informa sobre aspectos no tan consistentes, pero son fundamentales a la hora de interpretar la situación del grupo o centro que estamos valorando".

\section{Procedimiento}

La primera fase consistió en el estudio de la validez semántica del instrumento, para lo cual se hicieron modificaciones en el Autoinforme de la prueba INSSEBULL analizando cada ítem con un lingüista como lo sugieren Escobar-Pérez y CuervoMartínez (2008). Posteriormente, se solicitó la ayuda de 10 especialistas relacionados al ámbito escolar (9 psicólogos y un profesor) para validar las modificaciones realizadas previamente y analizar la validez semántica del instrumento mediante el criterio de jueces. Luego de recibir los resultados entregados por los expertos se aprobaron los cambios semánticos y se realizaron algunas modificaciones sintácticas por sugerencia de los jueces. Levantadas todas las observaciones se redactó el protocolo. Una vez que se contó con el protocolo final, se vio conveniente realizar el estudio piloto con ciertos estudiantes de los dos colegios de la muestra.

Previamente a las semanas de la evaluación se llevó a cabo diferentes reuniones con los padres de familia para explicarles los fines de la investigación y pedirles que firmasen el consentimiento informado. La evaluación tuvo una duración total de ocho semanas (cuatro semanas por colegio) y se realizó de manera individual, durante las dos horas del curso de tutoría.

A continuación, se procedió con el tratamiento estadístico de los datos según los objetivos planteados para la presente investigación y finalmente se cumplió con entregar los informes a cada colegio y realizar los talleres de convivencia saludable.

Las consideraciones éticas del presente estudio se estructuraron teniendo en cuenta los principios de la bioética. El principio de Autonomía se cumplió al informar detalladamente a los directores y estudiantes sobre el propósito del presente estudio. Además, se recalcó que la participación en este era totalmente voluntaria. Se solicitó el consentimiento informado a los padres y asentimiento a los estudiantes que colaboraron con la investigación. Respecto del principio de Beneficencia, se tuvo cuidado en la redacción de los ítems de la prueba, a fin de que estos no generen algún malestar en los participantes. Asimismo, se realizó un taller de convivencia con los tutores de los colegios sobre el tema de bullying 
y se entregaron tres sesiones para ser desarrolladas en las horas de tutoría con el objetivo de promover relaciones saludables entre los alumnos. Por último se capacitó a los psicólogos en la aplicación y corrección del Autoinforme para que este pueda seguir siendo usado dentro de los centros educativos. El principio de No Maleficencia se centra en no generar perjuicios a los estudiantes que formaron parte de la investigación. El uso de los resultados se restringió a los fines del presente estudio y a las necesidades de los colegios. Por último, el principio de Justicia en este estudio se mostró al garantizar que todo estudiante que contara con el permiso respectivo de sus padres tenía derecho a ser evaluado.

\section{Análisis de datos}

Para el procesamiento de los datos se usó el paquete estadístico IBM SPSS 25. Se comenzó con el análisis de la validez semántica, a través del criterio de jueces y el análisis cuantitativo de sus respuestas mediante el coeficiente V de Aiken (Escurra, 1988). Para la validez de constructo se optó por el análisis factorial. Para ello, en primer lugar, se determinaron los valores de las pruebas KMO y de Bartlett para saber si los datos admitían dicho análisis. En función de los resultados de estas pruebas, se llevó a cabo un análisis factorial confirmatorio (AFC) del modelo de ocho factores planteado por los autores del instrumento. Se consideró una carga factorial de al menos .30 para conservar un ítem (Morales, 2011; Nunnally, 1970). $\mathrm{Al}$ aplicar esta regla, uno de los factores quedó con un solo ítem por lo que se planteó la realización de un análisis factorial exploratorio (AFE). Con base en los resultados del AFE se procedió a un nuevo AFC excluyendo los ítems con pesos factoriales bajos. Para la evaluación del modelo factorial se calcularon los siguientes índices de ajuste: índice de bondad de ajuste (GFI), índice de bondad de ajuste corregido (AGFI), índice de ajuste normalizado (NFI) y el índice de ajuste relativo (RFI). Estos índices fueron valorados de acuerdo con los criterios de Manzano (2017). Concluida esta fase, se procedió a determinar la confiabilidad y los baremos con base en el modelo de seis factores. Para la confiabilidad, se evaluó la consistencia interna mediante el alfa de Cronbach y se calcularon las correlaciones ítem-test. Para establecer los baremos, se procedió a calcular los percentiles 50 y 80 , estableciendo tres niveles de interpretación. Previamente, se realizó la prueba U de Mann-Whitney para evaluar una posible diferencia significativa entre los dos colegios en las puntuaciones obtenidas.

\section{RESULTADOS}

\section{Validez Semántica}

Para establecer la validez semántica del Autoinforme de la prueba INSEBULL, cada ítem fue analizado y adaptado a los vocablos del español latino. Posteriormente, se revisaron los cambios con la ayuda de un lingüista y se procedió a someter el instrumento al análisis por parte de 10 jueces. Los resultados indicaron que los ítems: $2,3,4,6,7,8$, $9,10,11,12,13,14,15,16,17,18,19,20,21,22$, $23,24,26,27,28,29,32$ y 33 presentan un alto valor $\mathrm{V}$ de Aiken $(\mathrm{V} \geq 0,90)$ y por lo tanto, una buena validez semántica. Por otro lado, los ítems: 5, 25, 30 y 31 obtuvieron acuerdos de 8 de los 10 expertos $(\mathrm{V}=0,80)$. El ítem 34 fue aprobado por siete de los expertos $(\mathrm{V}=.70)$. Se procedió a modificar algunos aspectos de redacción en el ítem que obtuvo una $\mathrm{V}$ de Aiken menor a .80, de acuerdo a las recomendaciones de los jueces. Es necesario aclarar que el protocolo original de la prueba INSEBULL cuenta con 34 ítems, el primero de ellos es cualitativo. Sin embargo, para la presente investigación sólo se tomó en cuenta los ítems cuantitativos, es decir, todos los ítems del 2 al 34.

La dimensión Intimidación presenta valores $\mathrm{V}$ de Aiken altos que oscilan entre 0,80 y 1,00. Los ítems $20,21,22,24$ y 27 reportan una $\mathrm{V}=1,00$, lo que indica el acuerdo total de los jueces sobre la validez semántica de los mismos. Por otro lado, los ítems 19 y 23 presentan acuerdos de nueve de los jueces $(\mathrm{V}=$ .90). Finalmente, el ítem 25 obtuvo ocho acuerdos por parte de los jueces $(\mathrm{V}=.80)$. En la dimensión Victimización los ítems 10,11 y 13 presentan un acuerdo absoluto entre los 10 jueces, mientras que los ítems 12, 18 y 32 presentan el acuerdo de nueve de los jueces. En la dimensión Red Social, los ítems 2 y 4 alcanzaron una $\mathrm{V}=1,00$, el ítem 3 una $\mathrm{V}=0,90$ y el ítem 34, una $\mathrm{V}=0,70$. En cuanto a la dimensión Solución Moral, el valor de V para el ítem 29 fue 1,00, para el ítem 33 fue 0,90 y para los ítems 30 y 31 fue 0,80. En la dimensión Falta de Integración Social se hallaron valores de V de .90 para los ítems 6 y 7 y de 0,80 para el ítem 5. Asimismo, todos los ítems de la dimensión Constatación del Maltrato $(16,26$ y 28) y de la dimensión de Expresión de Temores Escolares (8 y 9) alcanzaron valores V de 1,00. Por último, en la dimensión de Identificación de los Participantes, los ítems 14 y 15 obtuvieron valores $\mathrm{V}$ de 1,00 y el ítem 17 , un valor $\mathrm{V}$ de 0,90 . 


\section{Análisis Factorial}

Previamente al análisis factorial se llevaron a cabo las pruebas KMO y de Esfericidad de Bartlett. En la primera se obtuvo un valor de .865 y en la segunda el resultado fue significativo $(p<0,05)$. Teniendo en cuenta estos resultados se consideró pertinente llevar a cabo el análisis factorial confirmatorio (AFC).

Para el AFC es común que se utilice el método de máxima verosimilitud; sin embargo, Hair et al (2007) mencionan que dicho método es adecuado cuando se cumple el supuesto de normalidad multivariante. Dado que en el presente estudio los datos no cuentan con una distribución normal (valor crítico 56,609 > 1,96 con un nivel de significación de 0,05 ), se usó el método de Mínimos Cuadrados de Libre Escala, el cual según Byrne (2010), es adecuado cuando la muestra es pequeña y el supuesto de normalidad en los datos no existe. Los valores obtenidos de los índices de ajuste fueron los siguientes: $\mathrm{GFI}=0,973, \mathrm{AGFI}=0,967, \mathrm{NFI}$ $=0,956$ y RFI $=0,951$. Todos estos valores indican un ajuste adecuado del modelo, considerando los criterios de valoración de Manzano (2017).

Al construir el modelo estructural presentado en la Figura 1, se observa que todos los ítems de las ocho dimensiones, presentaron cargas factoriales por encima de 0,30 , con excepción de los reactivos 2, 3, 6, 16, 17 y 28, los cuales debieron eliminarse. Sin embargo, al eliminar los ítems 16 y 28, el factor Constatación del Maltrato se quedó sólo con un reactivo, por lo que se consideró conveniente realizar un análisis factorial exploratorio con el objetivo de identificar una estructura más adecuada.

El análisis factorial exploratorio (AFE) con rotación Varimax convergió en seis factores, los cuales explicaron el $60 \%$ de la varianza. Se observó que se conservaron los factores de Intimidación, Victimización, Solución Moral, Identificación de los Participantes, Falta de Integración Social y Expresión de Temores Escolares; por otro lado, se eliminaron los factores Constatación del Maltrato y Red Social. Los ítems 26 y 34 cargaron en el factor Identificación de los Participantes y, por último, el ítem 4 pasó a formar parte de la dimensión Falta de Integración Social. En la Figura 2 se muestra el modelo estructural del instrumento con los seis factores hallados y en el que las dimensiones Intimidación, Victimización, Expresión de Temores Escolares, Solución Moral, Identificación de los Participantes y Falta de Integración Social cuentan con $8,6,2,4,4$ y 3 reactivos respectivamente. Posteriormente, se realizó un nuevo AFC con el método de Mínimos Cuadrados de Libre Escala y se calcularon los índices de ajuste del modelo de seis factores, habiendo eliminado los ítems 2, 3, 6, 16, 17 y 28. Los valores de los índices de ajuste fueron más altos que en el modelo de ocho factores: GFI $=0,980$, $\mathrm{AGFI}=0,975, \mathrm{NFI}=0,970$ y $\mathrm{RFI}=0,965$.

\section{Confiabilidad de Consistencia Interna}

La confiabilidad de las puntuaciones del Autoinforme de la prueba INSEBULL se evaluó mediante la consistencia interna. Previamente, se calcularon las correlaciones ítem-test para ver el comportamiento de cada reactivo. En la Tabla 1 se muestra las correlaciones ítem-test y el valor del alfa cuando se elimina cada elemento para el modelo de ocho factores. Se observa que los ítems que presentaron una carga factorial menor a .30 en el AFC (ítems 2, 3, 6, 16, 17 y 28) también presentan correlaciones ítem-total menores a .20, el cual, según Frías-Navarro (2019), es un valor poco adecuado. Esto refuerza la decisión de eliminar dichos reactivos y volver a calcular las correlaciones ítem-test sin estos ítems.

Por tanto, se procedió a un nuevo cálculo de las correlaciones ítem-test sin los ítems 2, 3, 6, 16, 17 y 28. El resultado se muestra en la Tabla 2. Se observa que los ítems 7, 29, 30, 31 y 33, pertenecientes a la dimensión Solución Moral, presentaron valores

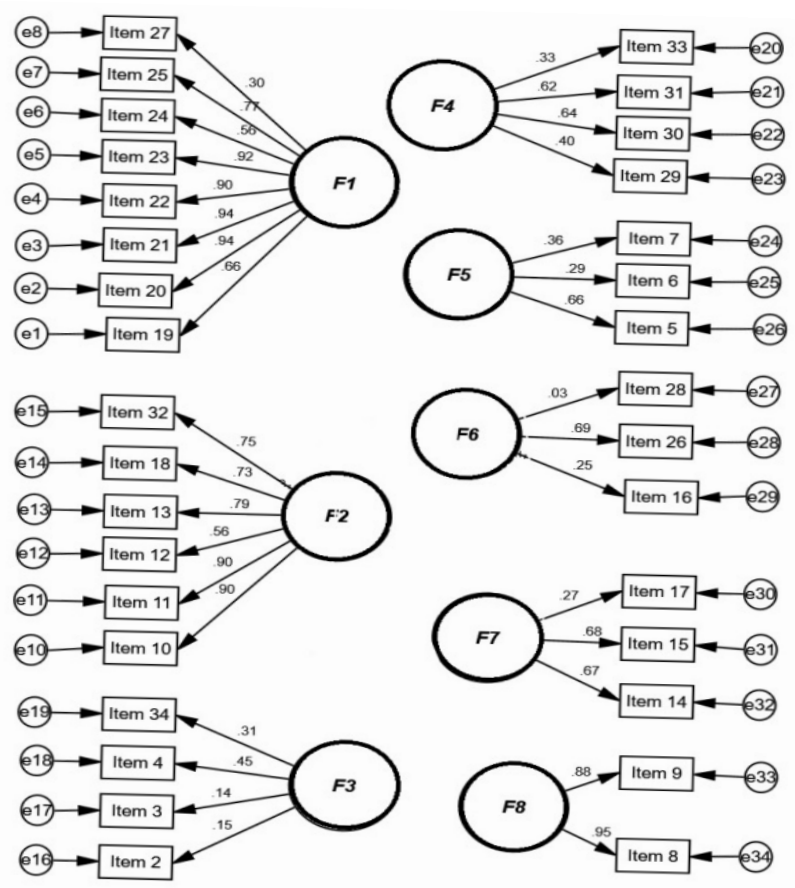

Figura 1. Modelo Estructural del Autoinforme del INSEBULL con Ocho Factores 


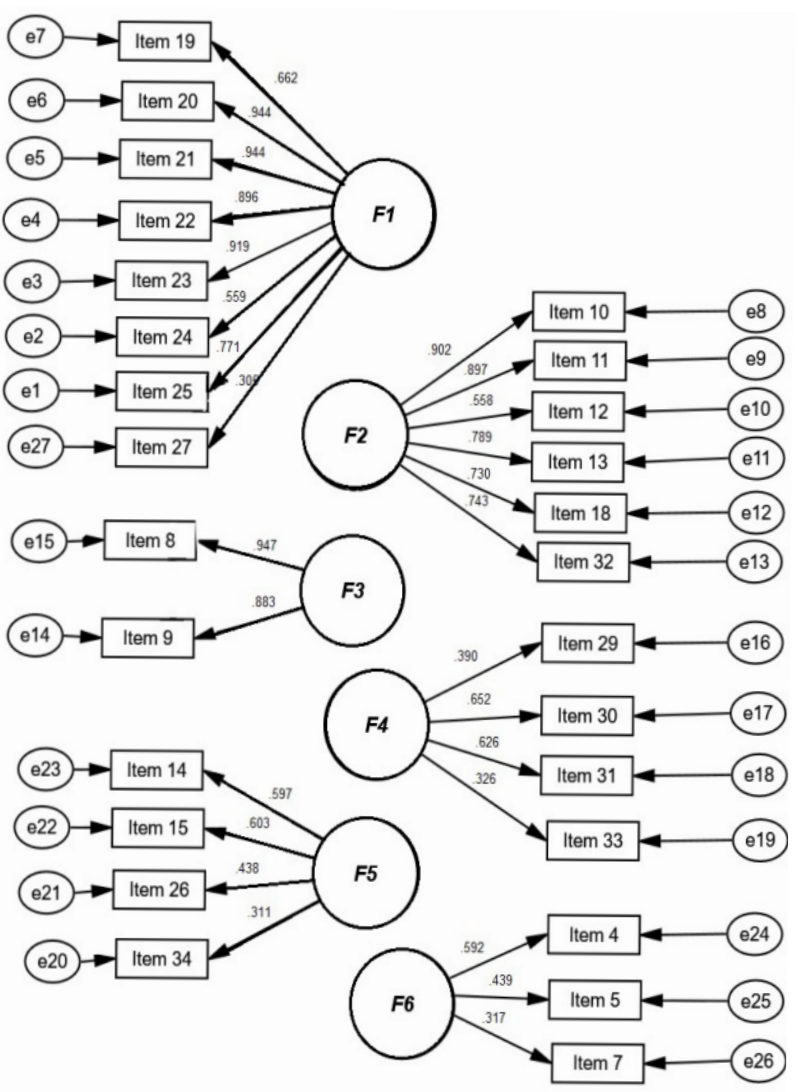

Figura 2. Modelo Estructural del Autoinforme del INSEBULL con Seis Factores

menores a .20; sin embargo, la eliminación de estos reactivos no tiene un impacto significativo en el incremento del alfa. Además estos ítems demostraron tener cargas factoriales significativas en el AFC, por lo que se decidió conservarlos, basando esta medida en lo sugerido por Blum et al. (2013), quienes indican que la decisión de eliminar un ítem debe estar fundamentada en más de solo un criterio desfavorable asociado a dicho reactivo. El alfa para la puntuación total también mejoró al eliminar los ítems con bajas correlaciones y baja carga factorial; de este modo, en la versión de 33 ítems el alfa fue 0,863 , en tanto que en la de 27 fue 0,873 .

Se procedió también a calcular los valores del alfa de Cronbach para cada uno de los factores del instrumento. Los resultados expuestos en la Tabla 3 indican que en los factores de Intimidación, Victimización y Expresión de Temores Escolares se observan altos niveles de consistencia interna. Por otro lado, las dimensiones Solución Moral, Falta de Integración Social e Identificación de Participantes presentan un alfa en un nivel débil.

\section{Baremos}

Antes de la elaboración de los baremos se evaluó si había o no diferencias entre las puntuaciones obtenidas de cada colegio. En el centro educativo estatal se obtuvo un puntaje total mínimo de 23 y un máximo de 124 , con una media de 57,45 y una desviación estándar de 24,41 . Por otro lado, en el centro educativo privado

Tabla 1. Primer Cálculo de Correlaciones Ítem-Test y Alfa de Cronbach

\begin{tabular}{|c|c|c|}
\hline Ítem & ritc & $\alpha$ \\
\hline 2 & 0,104 & 0,864 \\
\hline 3 & 0,101 & 0,865 \\
\hline 4 & 0,324 & 0,861 \\
\hline 5 & 0,267 & 0,862 \\
\hline 6 & 0,128 & 0,863 \\
\hline 7 & 0,154 & 0,863 \\
\hline 8 & 0,297 & 0,861 \\
\hline 9 & 0,266 & 0,862 \\
\hline 10 & 0,655 & 0,852 \\
\hline 11 & 0,643 & 0,852 \\
\hline 12 & 0,411 & 0,858 \\
\hline 13 & 0,563 & 0,853 \\
\hline 14 & 0,430 & 0,859 \\
\hline 15 & 0,421 & 0,859 \\
\hline 16 & 0,121 & 0,867 \\
\hline 17 & 0,101 & 0,863 \\
\hline 18 & 0,544 & 0,854 \\
\hline 19 & 0,549 & 0,854 \\
\hline 20 & 0,685 & 0,851 \\
\hline 21 & 0,685 & 0,851 \\
\hline 22 & 0,649 & 0,850 \\
\hline 23 & 0,693 & 0,851 \\
\hline 24 & 0,435 & 0,858 \\
\hline 25 & 0,574 & 0,854 \\
\hline 26 & 0,326 & 0,861 \\
\hline 27 & 0,259 & 0,862 \\
\hline 28 & 0,010 & 0,865 \\
\hline 29 & 0,149 & 0,865 \\
\hline 30 & 0,207 & 0,863 \\
\hline 31 & 0,201 & 0,863 \\
\hline 32 & 0,607 & 0,853 \\
\hline 33 & 0,106 & 0,869 \\
\hline 34 & 0,222 & 0,864 \\
\hline
\end{tabular}

Nota . ritc $=$ correlación elemento-total corregida, $\alpha=$ alfa de Cronbach si se elimina el elemento. 
Tabla 2. Segundo Cálculo de Correlaciones Ítem-Test y Alfa de Cronbach

\begin{tabular}{|c|c|c|}
\hline Ítem & ritc & $\alpha$ \\
\hline 4 & 0,314 & 0,871 \\
\hline 5 & 0,241 & 0,872 \\
\hline 7 & 0,149 & 0,873 \\
\hline 8 & 0,294 & 0,871 \\
\hline 9 & 0,255 & 0,872 \\
\hline 10 & 0,664 & 0,862 \\
\hline 11 & 0,654 & 0,862 \\
\hline 12 & 0,414 & 0,868 \\
\hline 13 & 0,565 & 0,864 \\
\hline 14 & 0,414 & 0,869 \\
\hline 15 & 0,413 & 0,869 \\
\hline 18 & 0,547 & 0,864 \\
\hline 19 & 0,558 & 0,864 \\
\hline 20 & 0,705 & 0,861 \\
\hline 21 & 0,705 & 0,861 \\
\hline 22 & 0,665 & 0,860 \\
\hline 23 & 0,707 & 0,860 \\
\hline 24 & 0,441 & 0,868 \\
\hline 25 & 0,593 & 0,864 \\
\hline 26 & 0,319 & 0,871 \\
\hline 27 & 0,268 & 0,872 \\
\hline 29 & 0,133 & 0,875 \\
\hline 30 & 0,192 & 0,874 \\
\hline 31 & 0,187 & 0,873 \\
\hline 32 & 0,610 & 0,863 \\
\hline 33 & 0,104 & 0,875 \\
\hline 34 & 0,222 & 0,875 \\
\hline
\end{tabular}

Nota . ritc $=$ correlación elemento- total corregida, $\alpha=$ alfa de Cronbach si se elimina el elemento.

se halló un puntaje total mínimo de 23 y un máximo de 126 con una media de 58,50 y una desviación estándar de 22,66. Los valores hallados en la prueba de MannWhitney $(U=25832,000 ; Z=-1,283 ; p=0,199)$ indicaron que no hay una diferencia estadísticamente significativa entre las puntuaciones totales obtenidas de ambos centros educativos, estatal y privado. Esto quiere decir que la percepción de los alumnos sobre el nivel de riesgo de verse envueltos en dinámicas de acoso escolar no difiere según el tipo de centro educativo en el que se encuentren estudiando.

A pesar de que no se encontró una diferencia significativa entre los puntajes totales de cada colegio,
Tabla 3. Alfa de Cronbach por Factor del Autoinforme de la Prueba INSEBULL

\begin{tabular}{lcc}
\hline \multicolumn{1}{c}{ Factor } & Alfa & n \\
\hline Intimidación & 0,909 & 8 \\
Victimización & 0,898 & 6 \\
Solución Moral & 0,507 & 4 \\
Falta de Integración Social & 0,401 & 3 \\
Identificación de Participantes & 0,514 & 4 \\
Expresión de Temores Escolares & 0,911 & 2 \\
\hline Nota. $\mathrm{n}=$ número de elementos.
\end{tabular}

se decidió establecer normas diferenciadas para interpretar de manera más cuidadosa los resultados de cada estudiante. En las Tablas 4 y 5 se muestran las puntuaciones correspondientes a los percentiles 50 y 80 para cada colegio. A partir de estos percentiles se definen tres categorías (bajo, medio y alto) para la interpretación de las puntuaciones.

\section{DISCUSIÓN}

El estudio de validez semántica, a través del método de criterio de jueces, identificó valores $\mathrm{V}$ de Aiken entre .80 y 1.00 para todos los ítems de la prueba, a excepción del ítem 34. Según Boluarte y Tamari (2017), los reactivos con valores V de Aiken superiores a .80 son adecuados y significativos, por lo que, en su mayoría, los ítems del Autoinforme de la prueba INSEBULL muestran una validez semántica adecuada. También Escurra (1988) señaló que un valor de $\mathrm{V}=.80$ es significativo al nivel de $p<.05$. El factor Identificación de los Participantes es el único que presenta un ítem (el 34) con siete acuerdos entre

Tabla 4. Percentiles para las Puntuaciones en las Dimensiones del Autoinforme del INSEBULL por Cada Centro Educativo

Dimensiones $\begin{gathered}\text { Colegio } \\ \text { estatal }\end{gathered} \quad \begin{gathered}\text { Colegio } \\ \text { privado }\end{gathered}$
$\begin{array}{llll}\text { pc } 50 & \text { pc } 80 & \text { pc } 50 & \text { pc } 80\end{array}$

\begin{tabular}{lcccc}
\hline Intimidación & 8 & 30 & 8 & 30 \\
Victimización & 9 & 25 & 9 & 26 \\
$\begin{array}{l}\text { Solución moral } \\
\begin{array}{l}\text { Falta de integración } \\
\text { social }\end{array}\end{array}$ & 5 & 13 & 7 & 10 \\
$\begin{array}{l}\text { Identificación de los } \\
\text { participantes }\end{array}$ & 15 & 19 & 17 & 20 \\
$\begin{array}{l}\text { Expresión de temores } \\
\text { escolares }\end{array}$ & 3 & 8 & 3 & 8 \\
Total & 50 & 85 & 55 & 81 \\
\hline
\end{tabular}


Tabla 5. Normas Percentiles para las Puntuaciones en los Factores del Autoinforme del INSEBULL por Cada Centro Educativo

\begin{tabular}{|c|c|c|c|c|c|c|}
\hline \multirow{3}{*}{ Factores } & \multicolumn{6}{|c|}{ Percentiles } \\
\hline & \multicolumn{2}{|c|}{$<\mathbf{5 0}$} & \multicolumn{2}{|c|}{$50-80$} & \multicolumn{2}{|c|}{$80<$} \\
\hline & $\mathbf{C E}$ & CP & CE & $\mathbf{C P}$ & CE & CP \\
\hline Intimidación & $0-8$ & $0-8$ & $9-30$ & $9-30$ & $31-42$ & $31-42$ \\
\hline Victimización & $0-9$ & $0-9$ & $10-25$ & $10-26$ & $26-36$ & $27-35$ \\
\hline Solución Moral & $0-8$ & $0-7$ & $9-13$ & $8-10$ & $14-21$ & $11-21$ \\
\hline $\begin{array}{l}\text { Falta de Integración } \\
\text { Social }\end{array}$ & $0-5$ & $0-5$ & $6-8$ & $6-8$ & $9-14$ & $9-12$ \\
\hline $\begin{array}{l}\text { Identificación de los } \\
\text { Participantes }\end{array}$ & $0-15$ & $0-17$ & $16-19$ & $18-20$ & $20-24$ & $21-29$ \\
\hline $\begin{array}{l}\text { Expresión de Temores } \\
\text { Escolares }\end{array}$ & $0-3$ & $0-3$ & $4-8$ & 4- 8 & $9-12$ & $9-12$ \\
\hline Total & $0-50$ & $0-55$ & $51-85$ & $56-81$ & $86-124$ & $82-126$ \\
\hline
\end{tabular}

los jueces $(\mathrm{V}=.70)$. Es necesario recalcar que, de los estudios psicométricos revisados sobre el Autoinforme de la prueba INSEBULL sólo Pinedo (2015) realizó la adaptación semántica con la ayuda de cinco expertos. En dicha investigación, al igual que en la presente, se modificaron los ítems $3,5,8$ y 26 y se hallaron valores $\mathrm{V}$ de Aiken en su mayoría superiores a .80; sin embargo, para que un ítem sea significativo en un grupo de cinco jueces se debe contar con el acuerdo de todos ellos (Escurra, 1988), a diferencia de un grupo de 10 jueces, en el que se solo se necesita el acuerdo de ocho de ellos para que el ítem sea significativo a un nivel de $p<.05$. Siguiendo estos criterios, se puede afirmar que los resultados de la presente investigación respecto de los valores hallados de $\mathrm{V}$, a pesar de ser similares a los del estudio de Pinedo (2015), poseen una mayor solidez estadística al haber incluido cinco jueces más.

En cuanto a la validez de constructo, mediante el AFC se puso a prueba el modelo original de ocho factores, el cual mostró buenos índices de ajuste, todos superiores a .90 , valores que según Cupani (2012), indican un nivel adecuado de congruencia entre las estructuras teóricas y los datos empíricos.
Sin embargo, se observó que los ítems 2, 3, 6, 16, 27 y 28 presentaban cargas factoriales por debajo de .30. Según Morales (2011), sí bien el punto de corte para determinar el nivel de la significación estadística del peso factorial puede variar según el tamaño de la muestra, nunca debe ser menor a .30. Tomando como base estos criterios, se decidió eliminar los ítems que no tenían un peso factorial mayor al valor indicado, y explorar una estructura más adecuada para el modelo. Posteriormente, se realizó un AFE y los datos convergieron en seis factores, los cuales explicaron el $60 \%$ de la varianza, porcentaje que es mayor al que se obtuvo en la prueba original con el modelo de ocho factores $(57,10 \%)$, sobre el que Avilés y Elices (2007, p.19) afirmaron era "adecuado para el tipo de instrumento que se analizaba: un Autoinforme". El modelo de seis factores encontrado en esta investigación difiere de los estudios realizados por Olaya et al. (2014), Pinedo (2015) y Rimaycuna (2015), quienes identificaron modelos de ocho, ocho y siete factores respectivamente. Por otro lado, es necesario recalcar que a pesar de la eliminación de dos factores, el modelo teórico no se ve afectado ya que Avilés y Elices (2007), autores de la prueba, mencionan que partieron de un modelo teórico de seis factores, 
en los que las dimensiones de Falta de Integración Social y Red Social estaban fusionadas, así como los factores de Constatación del Maltrato e Identificación de los Participantes. En los resultados de este estudio se pudo identificar que tras la eliminación de los ítems con cargas factoriales menores a .30, los resultados fueron congruentes con el modelo teórico previamente mencionado, dado que las dimensiones Red Social y Constatación del Maltrato se eliminaron y los ítems que las conformaban saturaron adecuadamente en los factores de Falta de Integración Social e Identificación de los Participantes, respectivamente. Además, el reactivo 34 pasó de la dimensión Red Social a la de Identificación de los Participantes, lo que tiene sentido teórico ya que este ítem tiene como objetivo identificar con qué rol de la dinámica de acoso escolar se identifica el estudiante. Finalmente, se realizó un último AFC con el propósito de evaluar el ajuste del modelo de seis factores, habiendo eliminado los ítems con cargas menores a .30. Se obtuvo que los valores de los índices no solo superaron satisfactoriamente los obtenidos para el modelo de ocho factores, sino que cumplen con criterios más estrictos como los planteados por autores como Hair et al. (2007) para quienes los valores más satisfactorios de estos índices de ajuste deben ser de al menos 0,95.

En cuanto a la consistencia interna, se observó que tras la eliminación de los ítems 2, 3, 6, 16, 17 y 28, los cuales no presentaban una buena correlación ítem-test $(<0,20)$ ni cargas factoriales adecuadas $(<0,30)$, el alfa de Cronbach se vio incrementado de 0,863 a 0,873 , valor que supera el valor mínimo $(0,70)$ planteado por Bojórquez et al. (2013) y Nunnally (1970) como adecuado. De igual manera, los valores de alfa en el presente estudio superan a los encontrados por Avilés y Elices (2007) y Pinedo (2015) quienes hallaron valores de .84 y .774 respectivamente. Por otro lado, se analizó la confiabilidad de cada uno de los seis factores identificados en esta investigación y se observó que las dimensiones Intimidación y Expresión de Temores Escolares presentan un alfa mayor a .90 por lo que tendrían un nivel "excelente" de consistencia interna según Frías-Navarro (2019). El factor Victimización $(\alpha=0,898)$ presenta un nivel "bueno" de consistencia interna, mientras que las dimensiones de Solución Moral, Identificación de los Participantes y Falta de Integración Social presentan una consistencia interna muy baja. Una de las razones que puede explicar estos resultados está relacionado con el número de ítems que estos factores contienen, ya que según Nunnally (1970), a más reactivos más consistencia interna y dichas dimensiones están formadas por pocos ítems en comparación con las de Intimidación y Victimización. Sin embargo, es importante recalcar que la dimensión de Expresión de Temores presenta una alta consistencia interna $(\alpha=0,911)$ a pesar de contar con solo dos ítems. Avilés y Elices (2007) hallaron en la versión original resultados similares a los del presente estudio para las dimensiones de Intimidación $(\alpha=0,90)$, Victimización $(\alpha=0,89)$ y Falta de integración social $(\alpha=.450)$. Mientras que para las dimensiones Solución Moral, Identificación de los Participantes y Expresión de Temores Escolares se hallaron en el presente estudio mejores niveles de alfa que en el estudio original, en el que dichos factores mostraron valores de 0,44 , 0,29 y 0,04 respectivamente. El caso de este último factor es particularmente resaltante ya que como se dijo anteriormente el valor del alfa de este factor es inusualmente alto, sobre todo en comparación al valor obtenido por Avilés y Elices (2007). En cuanto a los factores que presentan baja confiabilidad, Avilés y Elices (2007, p.21) mencionan que "si bien algunos factores muestran cierta debilidad, se recomienda usar dichas dimensiones a manera de indicios que aporten al manejo de la dificultad hallada" y afirman también que "a pesar de que ciertos factores informan sobre aspectos no tan consistentes, son fundamentales a la hora de interpretar la situación del grupo que se está valorando".

A pesar de que Avilés y Elices (2007) defiendan el uso de ciertos factores con baja consistencia interna debido a su contribución cualitativa en la evaluación e interpretación del acoso escolar; es necesario reconocer que puede que exista cierta debilidad intrínseca en dichas escalas, ya que en el presente estudio se refuerzan los datos sobre su bajo nivel de confiabilidad, específicamente en las escalas de Solución Moral, Identificación de los Participantes y Falta de Integración Social. Por otro lado, no se ha podido obtener más información sobre la confiabilidad de los factores en otras investigaciones, debido a que en primer lugar existen muy pocos estudios sobre este instrumento; y en segundo lugar porque ninguna de todas las investigaciones revisadas presenta datos sobre la consistencia interna de los factores. Por lo que, a pesar de que se reporten altos valores de alfa de Cronbach para la escala total, se desconoce en general el valor de este estadístico para cada dimensión, lo que deja un vacío en el análisis de este instrumento y arroja dudas sobre la idoneidad de su uso para la evaluación del bullying.

Se pudo observar que no existe una diferencia significativa en los puntajes totales según el tipo de 
colegio. Se esperaba que los resultados indicaran lo contrario ya que según las estadísticas del Ministerio de Educación del Perú (2019), del total de casos de bullying reportados en la plataforma Síseve entre setiembre del 2013 a mayo del 2019 , el $83 \%$ procedían de colegios públicos. Además del hecho de que las puntuaciones no son directamente comparables con el porcentaje de casos, también se debe tener en cuenta que, según Enríquez y Garzón (2015), los factores influyentes en la ocurrencia de bullying dentro de los colegios vendrían ser tres: el tamaño de la infraestructura, la organización interna y la presencia de profesores en distintas áreas. El centro educativo estatal donde se realizó parte del estudio tenía instalaciones pequeñas, por lo que los alumnos siempre estaban a la vista de los profesores; además, contaba con un "jefe de normas" cuyas funciones consistían en permanecer en los pasillos observando a los estudiantes, intervenir en posibles agresiones e informar a los tutores constantemente de la conducta de los alumnos. Se puede esperar que en los colegios estatales tienda a haber más casos de acoso escolar debido a que poseen gran cantidad de alumnos e instalaciones más amplias (Avilés, 2002); sin embargo, en el caso particular del presente estudio, puede ser que las características específicas mencionadas del colegio hayan actuado como factores protectores, gracias a los cuales se producen menos casos de acoso escolar en dicha institución. Finalmente, es conveniente ser precavido respecto al uso de los baremos presentados, dada la baja consistencia interna de varios de los factores del instrumento.

Es necesario seguir investigando sobre la consistencia interna de los factores para poder identificar con más precisión en qué grado el uso de este instrumento es prudente dentro de los contextos escolares, a pesar de la indicación de Avilés y Elices de que, en estas condiciones, es posible hasta cierto punto obtener información cualitativa sobre el fenómeno. En lo que respeta a la validez de constructo, se ha visto que el número de factores tiende a variar en los diferentes estudios, lo que podría indicar un modelo teórico no tan sólido, aspecto que sólo se puede seguir analizando a través de más estudios psicométricos detallados.

\section{Correspondencia:}

Cecilia Patricia Castro Chávarry

Correo electrónico: cecilia.castro.c@upch.pe

\section{REFERENCIAS BIBLIOGRÁFICAS}

Amemiya, I., Oliveros, M., \& Barrientos, A. (2009). Factores de riesgo de violencia escolar (bullying) severa en colegios privados de tres zonas de la sierra del Perú. Anales de la Facultad de Medicina, 70(4), 255-258.

Avilés, J (2002). Bullying: intimidación y maltrato entre el alumnado. Stee-Eilas.

Avilés, J. M., \& Elices, J. A. (2007). INSEBULL: Instrumentos para la evaluación del Bullying. CEPE.

Blum, D., Auné, S., Galibert, M., \& Attorresi, H. (2013). Criterios para la eliminación de ítems de un Test de Analogías Figurales. Summa Psicologica, 10(2), 4956.

Bojórquez, J., López, L., Hernández, M., \& Jiménez, E. (2013). Utilización del alfa de Cronbach para validar la confiabilidad de un instrumento de medición de satisfacción del estudiante en el uso del software Minitab. LACCEI. http://laccei.org/LACCEI2013Cancun/RefereedPapers/RP065.pdf

Boluarte, A., \& Tamari, K. (2017). Validez de contenido y confiabilidad inter-observadores de Escala Integral Calidad de Vida. Revista de Psicología (PUCP), 35(2),641-666.http://dx.doi.org/10.18800/psico.2017 02.009

Byrne, B. (2010). Structural equation modeling with AMOS. Taylor and Francis Group.

Castro-Morales, J. (2011). Acoso escolar: Bullying. Revista de Neuro-Psiquiatría, 74(2), 242-249. https:// doi.org/10.20453/rnp.v74i2.1681

Catena, A., Ramos, M., \& Trujillo, H. (2003). Análisis multivariado: un manual para investigadores. Biblioteca Nueva.

Ccoica, T. (2010). Bullying y funcionalidad familiar en una institución educativa del distrito de Comas. Universidad Nacional Federico Villarreal.

Cobián, C., Nizama, A., Ramos, D., \& Mayta, P. (2015). Medición y magnitud del bullying en Perú. Revista Peruana de Medicina Experimental y Salud Pública, 32(1), 196-197.

Comisión Nacional para el Desarrollo y Vida sin DrogasDEVIDA (2013). IV Estudio Nacional: prevención y consumo de drogas en estudiantes de secundaria). Informe. Regional.

Comisión Nacional para el Desarrollo y Vida sin Drogas. https://www.devida.gob.pe/documents/20182/97548/ Informes-Regionales-prevencion-consumo-drogasestudiantes-secundaria-2012.pdf

Córdova, A. J., Ramón, E. M., Jiménez, K., \& Cruz, C. J. (2012). Bullying y consumo de drogas. Revista de Psicología Universidad de Antioquia, 4(2), 21-48.

Cupani, M. (2012). Análisis de ecuaciones estructurales: conceptos, etapa de desarrollo y un ejemplo de aplicación. Revista Tesis, 2, 186-199.

Enríquez, M., \& Garzón, F. (2015). El acoso escolar. Saber, Ciencia y Libertad, 10(1), 219-233. 
Escobar-Pérez, J., \& Cuervo-Martínez, A. (2008). Validez de contenido y juicio de expertos: una aproximación a su utilización. Avances en Medición, 6, 27-36.

Escurra, L. (1988). Cuantificación de la validez de contenido por criterio de jueces. Revista de Psicología (PUCP), 6(2), 103-111.

Frías-Navarro, D. (2019). Apuntes de consistencia interna de las puntuaciones de un instrumento de medida. Universidad de Valencia. https:/www.uv.es/ friasnav/AlfaCronbach.pdf

Garaigordobil, M., \& Oñederra, J. A. (2010). La violencia entre iguales. Pirámide.

García, M. V., \& Ascensio, C. A. (2015). Bullying y violencia escolar: diferencias, similitudes, actores, consecuencias y origen. Revista Intercontinental de Psicología y Educación, 17(2), 9-38.

Hair, J. R., Anderson, R. E., Tatham, R. L., \& Black, W. C. (2007). Análisis multivariante. Pearson-Prentice Hall.

Joffre, V., García, G., Saldívia, A., Martínez, G., Lin, D., Quintanar, S., \& Villasana, A. (2011). Bullying en alumnos de secundaria. Características generales y factores asociados al riesgo. Boletín Médico del Hospital Infantil de México, 68(3), 193-202.

Manzano, A. P. (2017). Introducción a los modelos de ecuaciones estructurales. Investigación en Educación Médica, 7(25), 67-72. https://doi.org/10.1016/j. riem.2017.11.002

Ministerio de Educación (2019). Número de casos reportados en el portal SíseVe a nivel nacional. Ministerio de Educación del Perú. http://disde. minedu.gob.pe/handle/20.500.12799/6659

Morales, P. (2011). El análisis factorial en la construcción e interpretación de tests, escalas y Cuestionarios. Universidad Pontificia Comillas. http://www. upcomillas.es/personal/peter/investigacion/ AnalisisFactorial.pdf

Morales, P. (2012). Tamaño necesario de la muestra ¿Cuántos sujetos necesitamos? Universidad Pontificia Comillas. http://web.upcomillas.es/ personal/peter/investigacion/Tama\%floMuestra.pdf

Nunnally, J. (1970). Introducción a la medición psicológica. Paidós Ediciones.
Olaya, C. A., Pinto, N. G., \& Triana, Y. G. (2014). Validación del instrumento INSEBULL para su replicación en el contexto educativo de la ciudad de Villavicencio-Meta. [Proyecto de investigación para optar el título de psicólogo, Universidad Cooperativa de Colombia]. Repositorio institucional de la Universidad Cooperativa de Colombia. https://repository.ucc.edu.co/bitstream/20.500. 12494/5198/1/2014_validacion_instrumento insebull.pdf

Olweus, D. (1998). Conductas de acoso y amenaza entre escolares. Morata.

Ortega, R., Mora-Merchán, J. A., \& Mora, J. (2006) Cuestionario sobre intimidación y maltrato entre iguales. https://www.uco.es/laecovi/img/recursos/ nrKribUW8NHWlLe.pdf

Pinedo, O. E. (2015). Propiedades psicométricas del instrumento para la evaluación del Bullying INSEBULL en alumnos de secundaria. Revista de Investigación de Estudiantes de Psicología, 1, 31-50. http://revistas.ucv.edu.pe/index.php/JANG/ article/view/482

Piñuel, I., \& Oñate, A. (2005). Autotest Cisneros de Acoso escolar. ACP Edición. https://kupdf.net/ download/manual-de-cisnerosdoc_5cc32421e2b6f5e e516ba4bf_pdf

Rimaycuna, J. (2015). Nivel de acoso escolar en alumnos del $3^{\circ}$ al $5^{\circ}$ grado de secundaria en una institución educativa nacional de la ciudad de Chiclayo. [Informe de investigación para obtener el título de licenciado en psicología, Universidad Privada Juan Mejía Baca]. Repositorio institucional de la Universidad Privada Juan Mejía Baca. http:// repositorio.umb.edu.pe/handle/UMB/27

Sáenz, M (2010). Sintomatología depresiva y acoso escolar en un grupo de adolescentes escolares. Pontificia Universidad Católica del Perú.

Wolf, C., \& Esteffan, K. (2008). Bullying: una mirada desde la salud pública. Revista Chilena de Salud Pública, 12(3), 181-187.

Recibido: 01/11/2020

Aceptado: 01/12/2020 